\title{
Article \\ A Human Pan-Cancer System Analysis of Procollagen-Lysine, 2-Oxoglutarate 5-Dioxygenase 3 (PLOD3)
}

\author{
Siming Gong ${ }^{1}$, Yingjuan Duan ${ }^{2}$, Changwu Wu ${ }^{1, *}$, Georg Osterhoff ${ }^{3}$, Nikolas Schopow ${ }^{1,3,+}$ \\ and Sonja Kallendrusch ${ }^{1,+}$ \\ 1 Institute of Anatomy, University of Leipzig, Liebigstraße 13, 04103 Leipzig, Germany; \\ siminggong23@gmail.com (S.G.); nikolas.schopow@medizin.uni-leipzig.de (N.S.); \\ sonja.kallendrusch@medizin.uni-leipzig.de (S.K.) \\ 2 Faculty of Chemistry and Mineralogy, University of Leipzig, 04103 Leipzig, Germany; \\ yingjuan0103@gmail.com \\ 3 Sarcoma Center, Department of Orthopedics, Trauma and Plastic Surgery, University Hospital Leipzig, \\ 04103 Leipzig, Germany; georg.osterhoff@medizin.uni-leipzig.de \\ * Correspondence: wuchangwu93@gmail.com \\ + These authors contributed equally to this work and share the last authorship.
}

Citation: Gong, S.; Duan, Y.; Wu, C.; Osterhoff, G.; Schopow, N.;

Kallendrusch, S. A Human

Pan-Cancer System Analysis of Procollagen-Lysine, 2-Oxoglutarate 5-Dioxygenase 3 (PLOD3). Int. J. Mol. Sci. 2021, 22, 9903. https://doi.org/ $10.3390 /$ ijms 22189903

Academic Editor: Marco Falasca

Received: 29 July 2021

Accepted: 11 September 2021

Published: 14 September 2021

Publisher's Note: MDPI stays neutral with regard to jurisdictional claims in published maps and institutional affiliations.

Copyright: () 2021 by the authors. Licensee MDPI, Basel, Switzerland. This article is an open access article distributed under the terms and conditions of the Creative Commons Attribution (CC BY) license (https:// creativecommons.org/licenses/by/ $4.0 /)$.

\begin{abstract}
The overexpression of the enzymes involved in the degradation of procollagen lysine is correlated with various tumor entities. Procollagen-lysine, 2-oxoglutarate 5-dioxygenase 3 (PLOD3) expression was found to be correlated to the progression and migration of cancer cells in gastric, lung and prostate cancer. Here, we analyzed the gene expression, protein expression, and the clinical parameters of survival across 33 cancers based on the Clinical Proteomic Tumor Analysis Consortium (CPTAC), function annotation of the mammalian genome 5 (FANTOM5), Gene Expression Omnibus (GEO), Genotype-Tissue Expression (GTEx), Human Protein Atlas (HPA) and The Cancer Genome Atlas (TCGA) databases. Genetic alteration, immune infiltration and relevant cellular pathways were analyzed in detail. PLOD3 expression negatively correlated with survival periods and the infiltration level of $\mathrm{CD}^{+} \mathrm{T}$ cells, but positively correlated to the infiltration of cancer associated fibroblasts in diverse cancers. Immunohistochemistry in colon carcinomas, glioblastomas, and soft tissue sarcomas further confirm PLOD 3 expression in human cancer tissue. Moreover, amplification and mutation accounted for the largest proportion in esophageal adenocarcinoma and uterine corpus endometrial carcinoma, respectively; the copy number alteration of PLOD3 appeared in all cancers from TCGA; and molecular mechanisms further proved the effect of PLOD3 on tumorigenesis. In particular, PLOD3 expression appears to have a tumor immunological effect, and is related to multiple immune cells. Furthermore, it is also associated with tumor mutation burden and microsatellite instability in various tumors. PLOD3 acts as an inducer of various cancers, and it could be a potential biomarker for prognosis and targeted treatment.
\end{abstract}

Keywords: PLOD3; pan-cancer; prognosis; tumor; immune infiltration; enrichment analysis; immunotherapy; big data

\section{Introduction}

The expression of procollagen-lysine, 2-oxoglutarate 5-dioxygenase 3 (PLOD3) is essential for the biosynthesis of collagen and gene mutations thereof, and are related to disorders of the connective tissue [1-3]. Clinical related mutations with reduced expressions of the PLOD family are associated with the Ehlers-Danlos and Bruck syndromes [4-6]. PLOD3 alterations during tissue repair might, thus, lead to epithelial-mesenchymal transition (EMT). This process describes the transformation of stable epithelial cells undertaking phenotypic transformation. Consequently, cell to cell adherence as well as cell polarity are altered, gaining the ability to migrate and invade novel territories. EMT is a key process in wound healing, tissue repair and, also, in the development of a variety of cancers [7-9]. 
Among others, fibroblasts, chondroblasts and osteoblasts produce procollagen. Many post-translational modifications are necessary to generate collagens, which build a triplehelical molecule. PLOD3 belongs to a family of enzymes that is able to catalyze the lysine hydroxylation of procollagen using $\mathrm{Fe}^{2+}$. However, an upregulation of PLOD3 was recently found in fibrosis and solid tumors with poor prognosis $[3,10,11]$.

Current literature suggests a strong relation between collagen modulation and cancer $[3,10]$. In this pan-cancer system analysis, PLOD3 expression is being investigated in a broad variety of human solid tumor data, available within the Clinical Proteomic Tumor Analysis Consortium (CPTAC), function annotation of the mammalian genome 5 (FANTOM5), Gene Expression Omnibus (GEO), Genotype-Tissue Expression (GTEx), Human Protein Atlas (HPA) and The Cancer Genome Atlas (TCGA) databases.

The study analyzes gene alteration information, protein expression, prognostic ability and gene immune analysis of the PLOD3 of more than 10,000 samples from several comprehensive databases. Furthermore, we performed an enrichment analysis of related genes to investigate the potential role and molecular mechanism of PLOD3.

This study aims to understand the role and potential mechanism of PLOD3 in the process of oncogenesis of 33 tumors in humans.

\section{Results}

\subsection{Experiment Setup and Genetic Alteration Analysis Data}

Figure 1 shows the setup of this study for the tumors, including: adrenocortical carcinoma (ACC), breast invasive carcinoma (BRCA), bladder urothelial carcinoma (BLCA), colon adenocarcinoma (COAD), cholangiocarcinoma (CHOL), cervical squamous cell carcinoma and endocervical adenocarcinoma (CESC), lymphoid neoplasm diffuse large B-cell lymphoma (DLBC), esophageal carcinoma (ESCA), glioblastoma multiforme (GBM), head and neck squamous cell carcinoma (HNSC), kidney chromophobe (KICH), kidney renal clear cell carcinoma (KIRC), kidney renal papillary cell carcinoma (KIRP), liver hepatocellular carcinoma (LIHC), lower grade glioma (LGG), acute myeloid leukemia (LAML), lung adenocarcinoma (LUAD), lung squamous cell carcinoma (LUSC), mesothelioma (MESO), ovarian serous cystadenocarcinoma (OV), prostate adenocarcinoma (PRAD), pheochromocytoma and paraganglioma (PCPG), pancreatic adenocarcinoma (PAAD), rectum adenocarcinoma (READ), sarcoma (SARC), stomach adenocarcinoma (STAD), skin cutaneous melanoma (SKCM), thymoma (THYM), thyroid carcinoma (THCA), testicular germ cell tumors (TGCT), uterine carcinosarcoma (UCS), uterine corpus endometrial carcinoma (UCEC) and uveal melanoma (UVM).

Figure 2a illustrates the mutation, amplification, deep deletion and multiple alterations frequency of the PLOD3 gene and the copy number alteration (CNA) across different cancers. The CNA of PLOD3 occurred in all of these cancers except KICH, THYM and UVM. In ESCA, amplification accounted for the largest proportion $(8.2 \%)$, while in UCEC and SKCM, the largest part was mutation $(7.9 \%$ and $3.6 \%)$.

The diagram of mutation sites, additionally, explains the different types and the case number. Missense was the most common type of mutation with the number of 123, followed by truncating mutation with the number 26 (Figure $2 b$ ). 


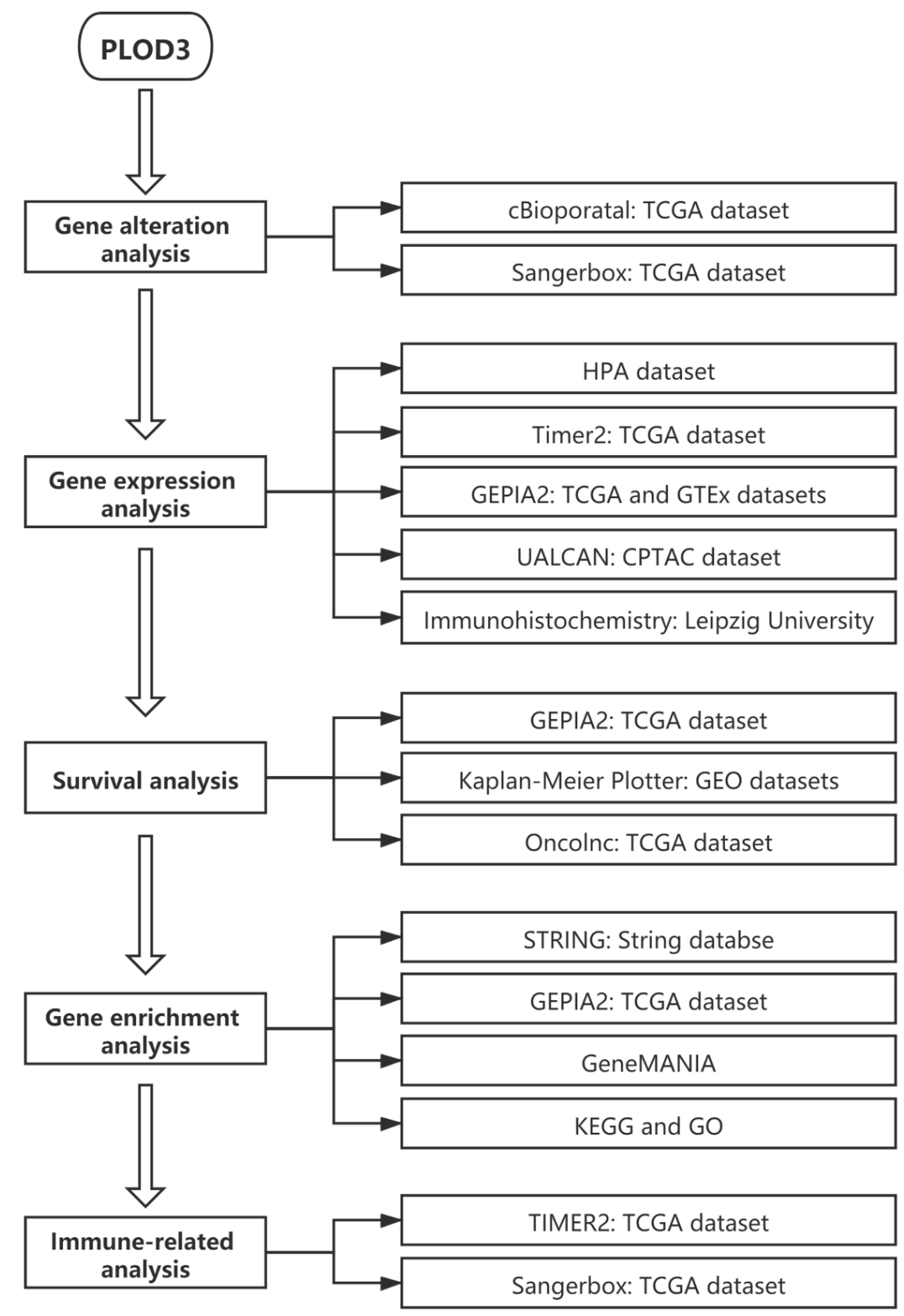

Figure 1. Setup of the integrative and comprehensive pan-cancer analysis of PLOD3. 
a

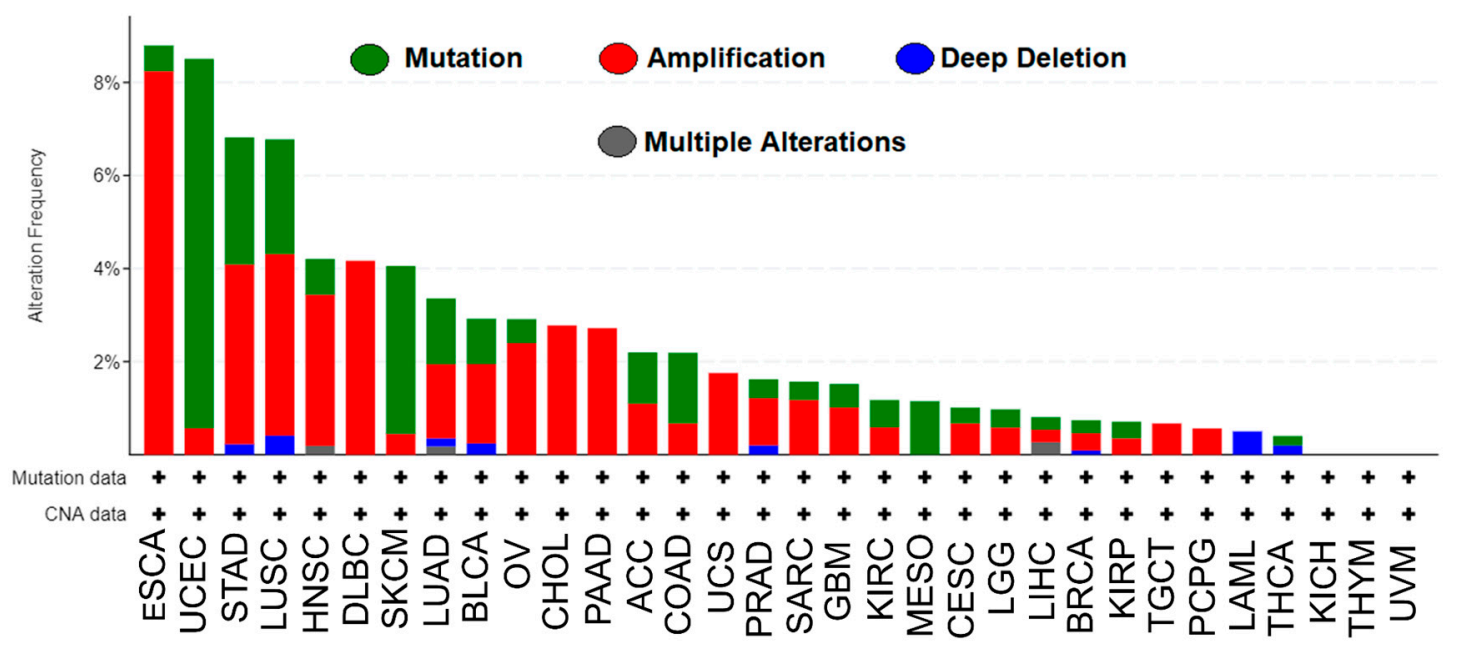

b

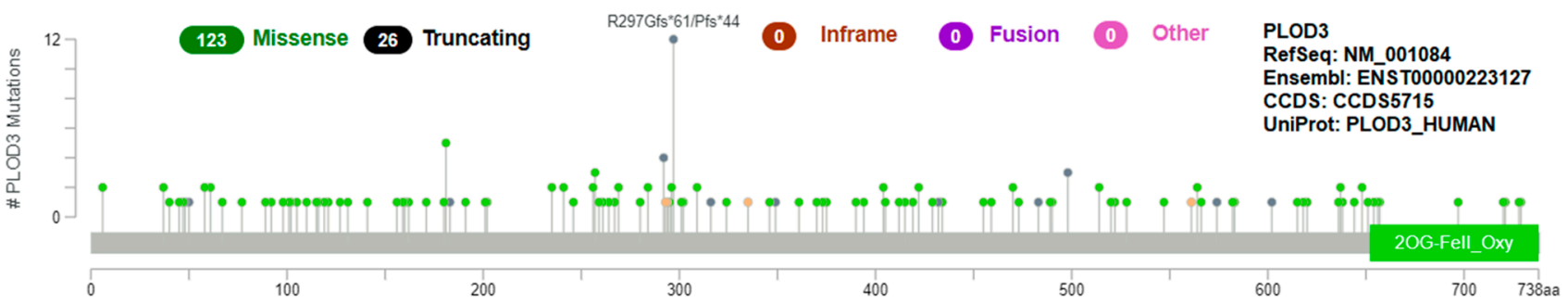

Figure 2. PLOD3 mutation feature in different tumors of TCGA. The alteration frequency and the type is shown in (a); The mutation types, sites and frequency of PLOD3 genetic alteration are displayed in (b).

\subsection{Gene Expression Analysis Data}

In the beginning, the expression level of PLOD3 in healthy tissue were analyzed based on GTEx, HPA, and FANTOM5. Supplementary Figure S1a illustrates that PLOD3 expressions are highest in human liver, vaginal and midbrain tissue. However, PLOD3 is expressed in most origins but showed low RNA specificity between different tissues. This low RNA specificity was also detected in the variable blood cells, based on a combination of the three datasets (Supplementary Figure S1b).

The expression of the PLOD3 gene was analyzed in the context of the TCGA database. Figure 3a shows that PLOD3 expression is higher in tumor tissues than in adjacent normal tissues across different cancers, such as BLCA, BRCA and CHOL (all $p<0.05)$. However, some tumor entities (complete ACC, DLBC, LGG, limited PCPG) are missing sufficient information on healthy origin tissues in the respective dataset of TCGA. 


\section{a TCGA dataset}

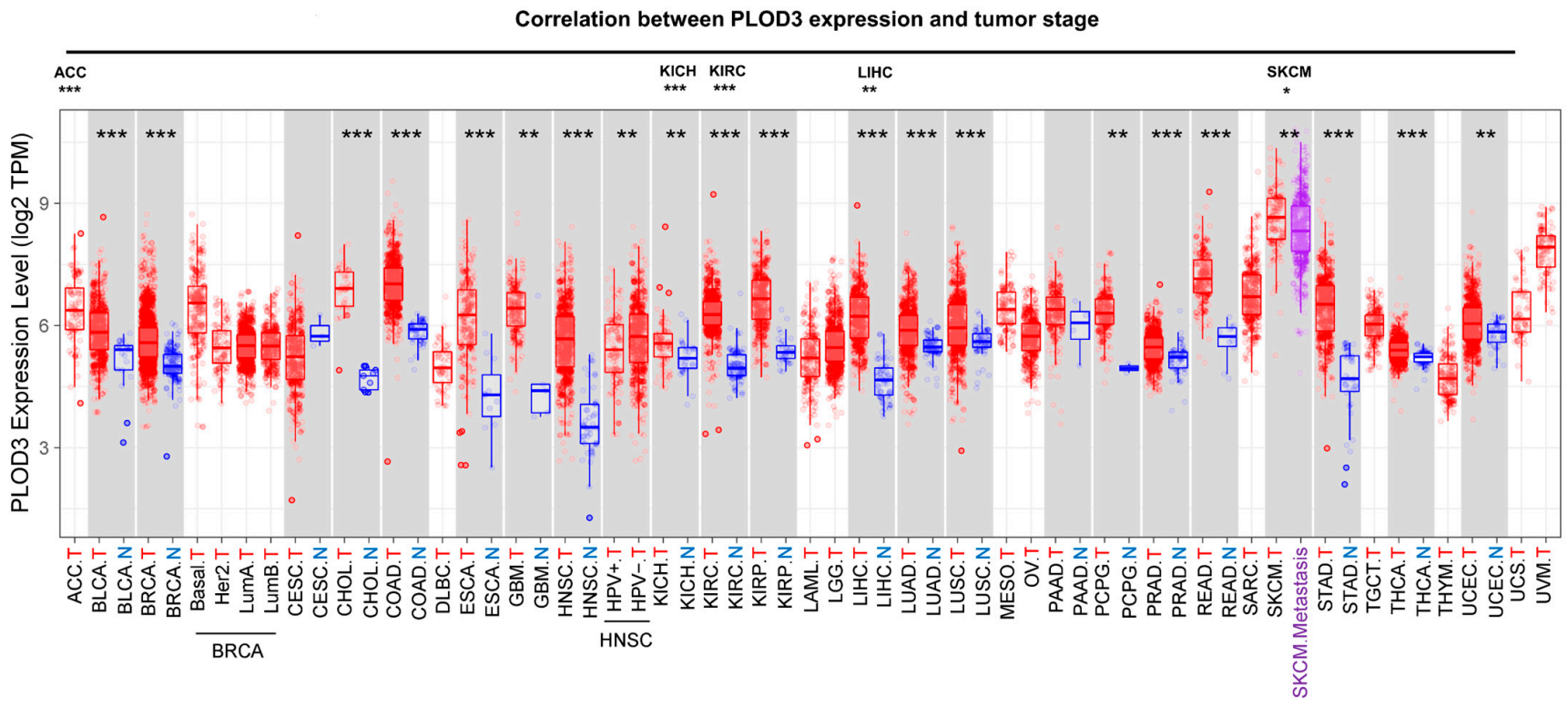

\section{b CPTAC dataset}
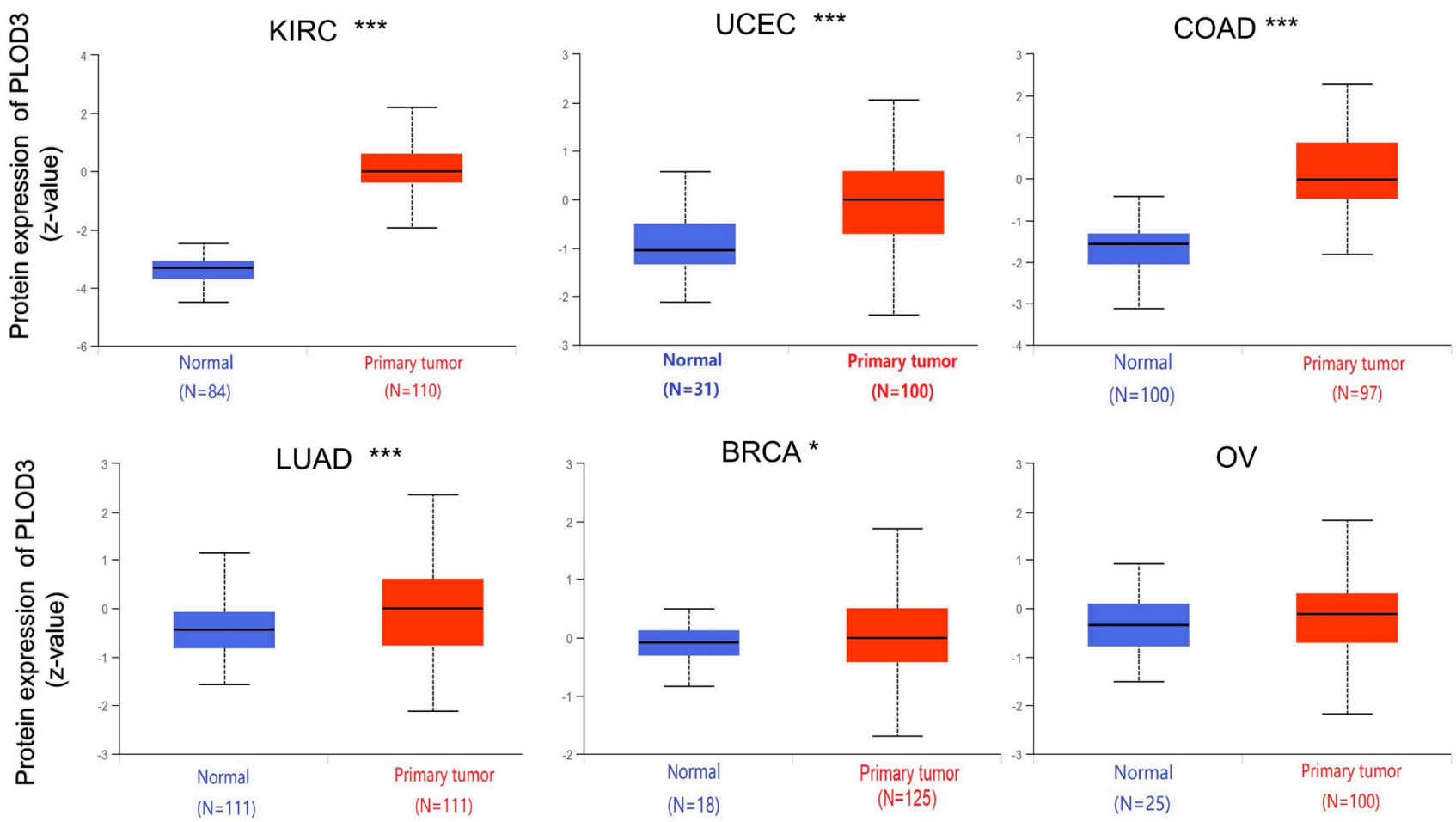

Figure 3. PLOD3 expression in different cancers. (a) The PLOD3 expression level in different cancers or specific cancer subtypes. N-normal and T-tumor tissue; (b) The PLOD3 total protein expression between tumor tissue and corresponding normal tissue according to the CPTAC dataset. ${ }^{*} p<0.05 ;{ }^{* *} p<0.01 ;{ }^{* *} p<0.001$.

Compared to normal tissue samples from the GTEx dataset serving as controls, PLOD3 expression is higher in some tumors, such as ACC, DLBC and LGG (Supplementary Figure S2a), but no significant difference was seen for LAML, OV, SARC and UCS (Supplementary Figure S2b).

In the CPTAC dataset, PLOD3 total protein expresses higher in primary cancers than in normal tissues for COAD, KIRC, UCEC, BRCA and LUAD, while the expression in ovarian cancer shows no increased protein expression (Figure 3). 
Representative PLOD3 immunohistochemistry staining is shown in Figure 4. It illustrates that the expression of PLOD3 from patient derived COAD, GBM, and SARC tissue was higher compared to the expression in corresponding healthy human tissue. In particular, the staining shows PLOD3 expression in the stromal cell populations of the TME, not of the tumor cells themselves.
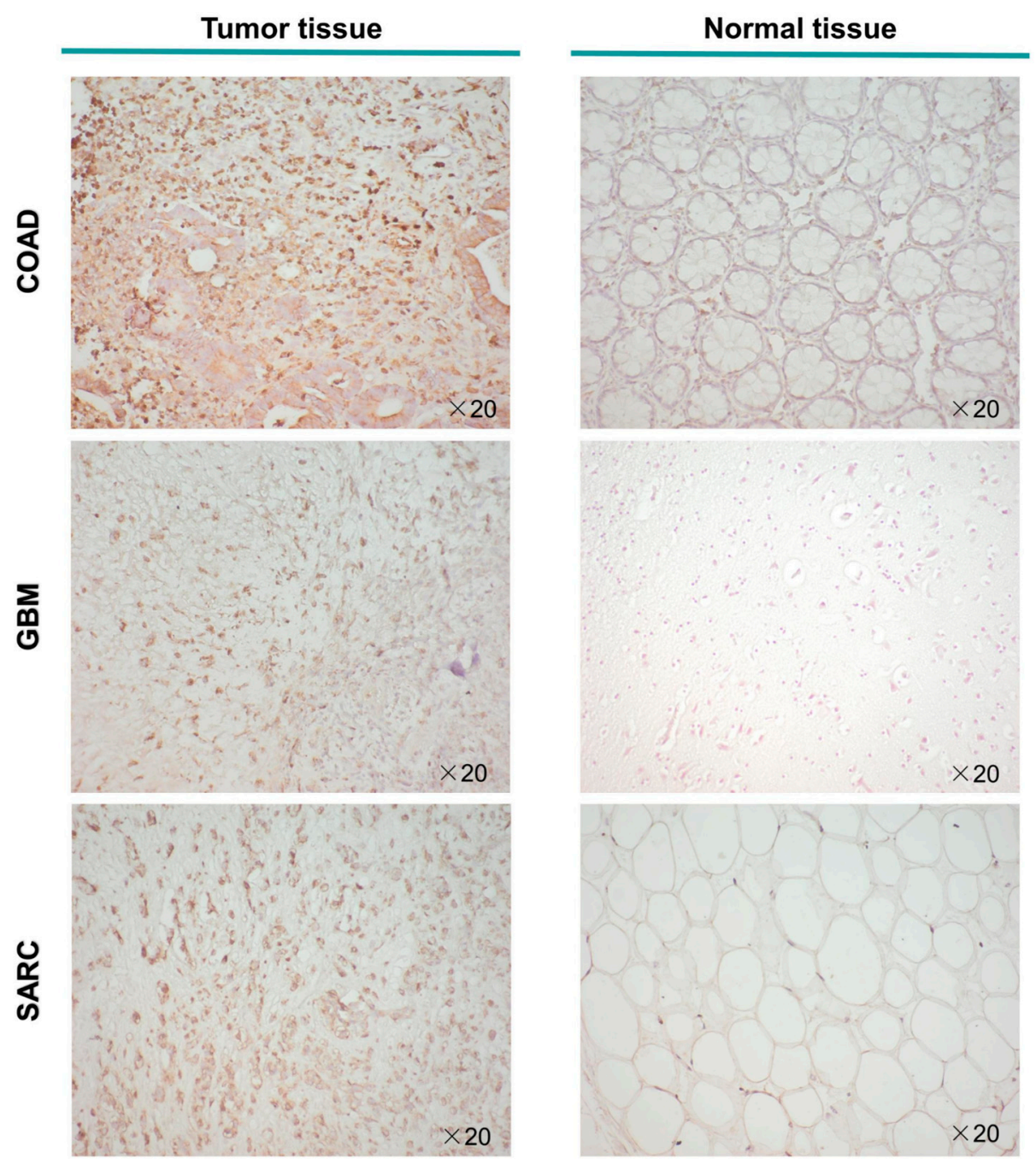

Figure 4. Immunohistochemical staining using patient derived tissue: PLOD3 expression in human COAD, GBM and SARC compared with corelating normal tissue (colon, brain, and adipose tissue).

Additionally, the expression of PLOD3 was related to pathological stages in ACC, $\mathrm{KICH}, \mathrm{KIRC}$, LIHC and SKCM, but not significantly related to the stages of other cancers (Supplementary Figure S3).

\subsection{Survival Analysis Data}

We separated the TCGA database into two groups. High and low PLOD3 expression and tumor prognosis was compared. Figure 5 shows overall survival (OS) and disease-free survival (DFS) for different kinds of tumors. 


\section{a Overall Survival}
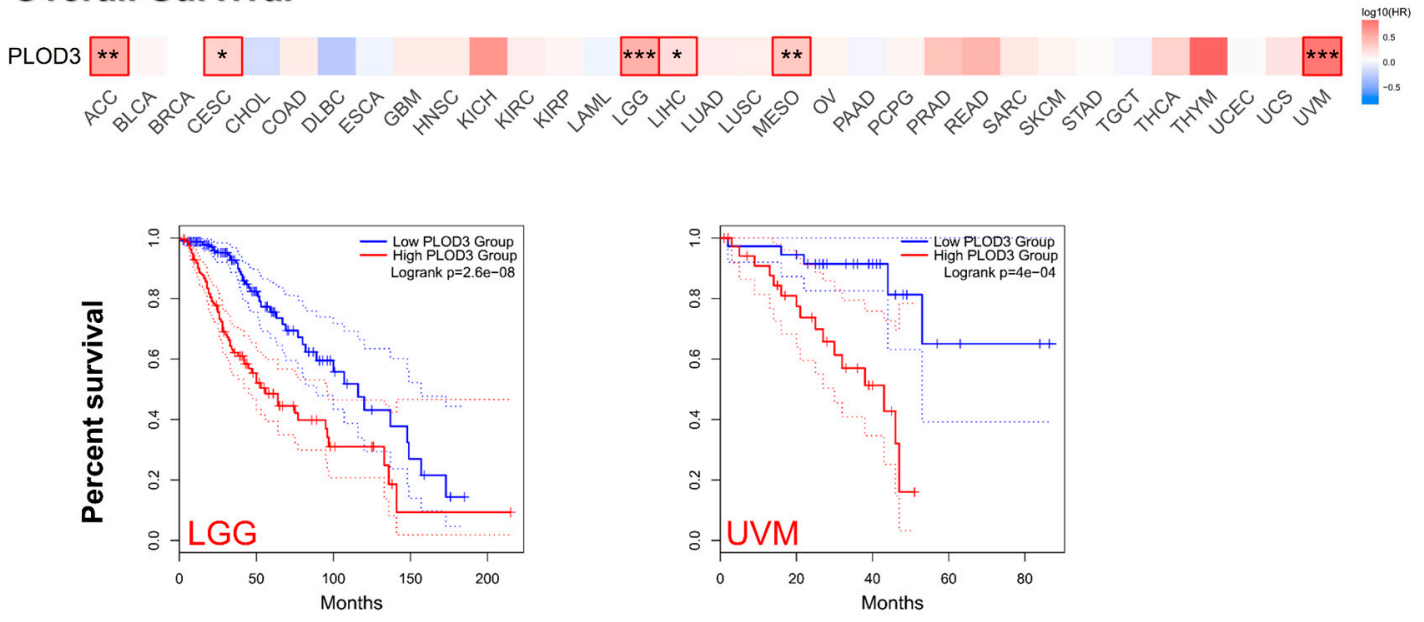

b Disease Free Survival
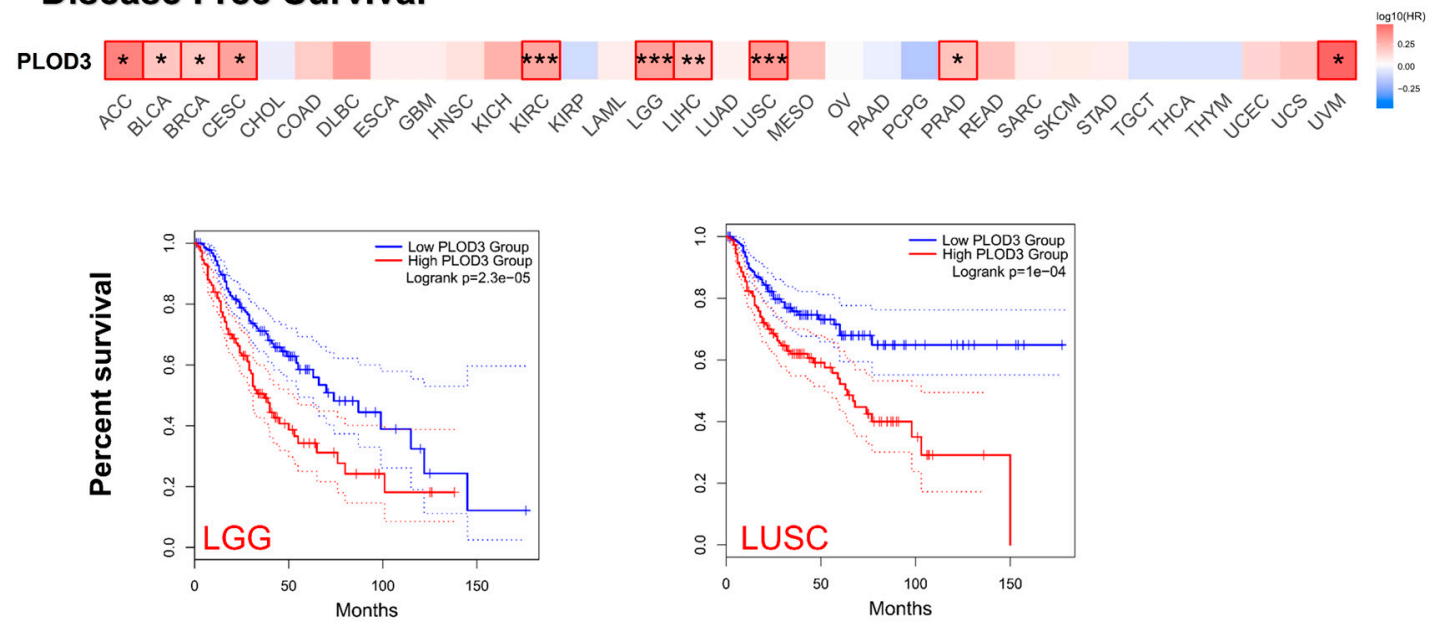

Figure 5. Relation between PLOD3 expression and survival prognosis of different cancers in TCGA. GEPIA2 tool was used to obtain the OS (a) and DFS (b) analyses of different tumors in TCGA. The survival maps and Kaplan-Meier curves are shown. Dotted lines: $95 \%$ confidence interval. ${ }^{*} p<0.05,{ }^{* *} p<0.01,{ }^{* * *} p<0.001$.

The survival map shows the relation between PLOD3 expression and OS. A high expression of PLOD3 was associated with poor OS in some cancers, namely, in ACC, CESC, LGG, LIHC, MESO and UVM (all $p<0.05$, Figure $5 \mathrm{a}$ ). Figure $5 \mathrm{~b}$ demonstrates the negative correlation between PLOD3 expression and DFS in ACC, BLCA, BRCA, CESC, KIRC, LGG, LIHC, LUSC, PRAD and UVM (all $p<0.05$ ). In the Kaplan-Meier curves, the correlation between the high and low expression groups was exemplified for OS of LGG and UVM and for DFS of LGG and LUSC (Figure 5).

Supplementary Figures S4 and S5 also illustrate that the high expression of PLOD3 was associated with poor prognosis in cancers such as KIRC, LUAD, OV and READ.

PLOD3 expression is negatively correlated with different prognosis periods: e.g., breast cancer: overall, relapse free and distant metastasis free survival; or lung cancer: first progression, overall and postprogression survival (all $p<0.05$, Supplementary Figure S6 also shows gastric, liver and ovarian cancer).

\subsection{Analysis of Related Genes Network}

For a deeper exploration of the potential mechanisms behind PLOD3 in oncogenesis, we observed the top 50 PLOD3-interacting proteins and carried out a protein-protein interaction network (Figure 6a) and obtained the positive relation of PLOD3 with top 100 PLOD3 correlated genes. The top 5 were: SRY-Box Transcription Factor 10 (SOX10) 
$(\mathrm{R}=0.61)$, Actin Related Protein 2/3 Complex Subunit 1B (ARPC1B) $(\mathrm{R}=0.59)$, Spermatidspecific linker histone H1-like protein (HILS1) $(\mathrm{R}=0.56)$, Parvin Beta (PARVB) $(\mathrm{R}=0.55)$ and Bridging Integrator 3 (BIN3) $(\mathrm{R}=0.55)$ (Figure $6 \mathrm{~b}$, all $p<0.05)$.

a

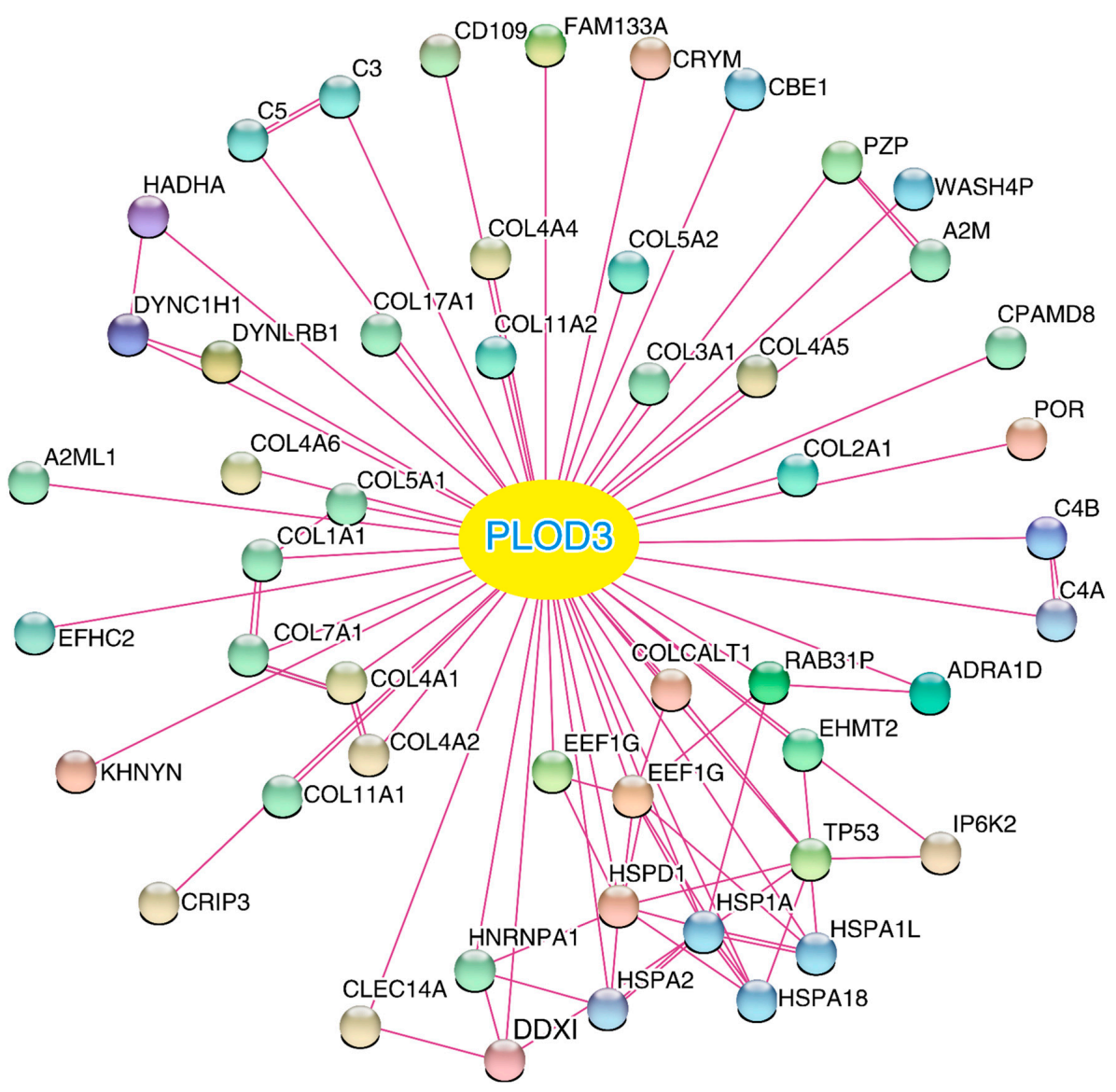

b
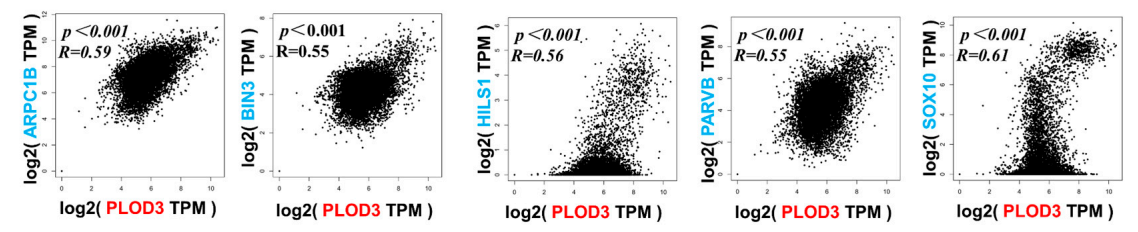

C

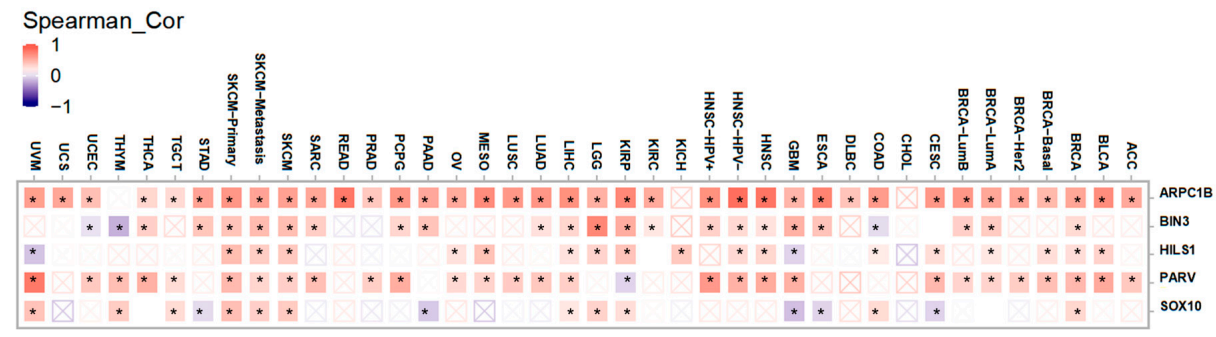

Figure 6. PLOD3 related gene enrichment analysis. (a) STRING tool was used to obtain the available, experimentally determined PLOD3-related proteins; (b) The GEPIA2 approach was employed to obtain the top 100 PLOD3-correlated genes in TCGA database and the relation between PLOD3 and selected targeting gene expressions were analyzed. The top five genes are shown; (c) The corresponding heatmap data in all investigated cancer types are displayed. ${ }^{*} p<0.05$.

Figure $6 \mathrm{c}$ presents the positive relation between PLOD3 expression level, with ARPC1B, HILS1, PARVB and BIN3 in most cancers from TCGA database, and a positive correlation with SOX10 in some of the cancers. 


\subsection{KEGG Pathway and GO Enrichment Analysis Data}

Kyoto Encyclopedia of Genes and Genomes (KEGG) pathway analysis shows that the expression of PLOD3 is associated with many factors, and the PLOD3 gene could be involved in oncogenesis and cancers metastasis by "protein digestion and absorption", "focal adhesion" and "small cell lung cancer" (Figure 7a).
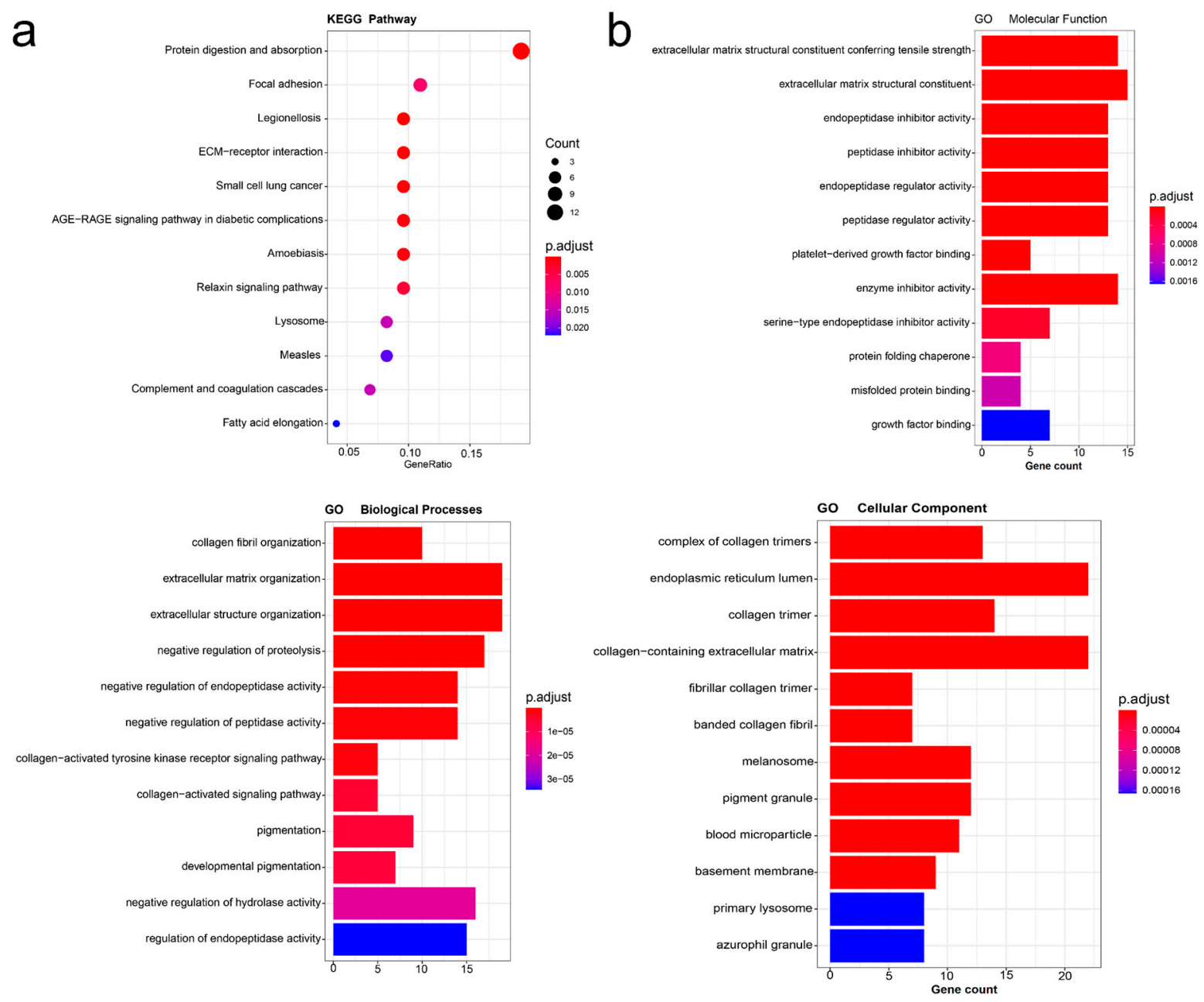

Figure 7. The enrichment analysis of PLOD3 and most frequent neighboring genes. (a) KEGG pathway analysis was obtained according to PLOD3-binding and interacted genes; (b) Bar plot of GO enrichment in cellular component terms, biological process terms, and molecular function terms.

Gene Ontology (GO) enrichment analysis (Figure 7b) indicated that PLOD3 and related genes were mainly associated with cellular components, such as endoplasmic reticulum lumen, biological processes such as extracellular matrix organization, collagen-activated tyrosine kinase receptor signaling pathway and regulation of endopeptidase activity, and molecular function, e.g., protein folding chaperone and misfolded protein binding.

\subsection{Immune Infiltration Analysis Data}

The heatmap shows correlations between PLOD3 expression and cancer associated fibroblasts (CAF) across diverse cancers of TCGA database. These analyses, based on the algorithms EPIC, MCPCOUNTER and TIDE, and the correlations with the same trend in all three were considered to be plausible. The expression of PLOD3 is positively related to CAF for most of the mentioned cancers, such as BLCA, SARC and CESC (Figure 8a). The scatter plot also illustrates the positive relation of PLOD3 expression and CAF in the 
aforementioned cancers. Based on the algorithms, cancers with the strongest correlation were shown (Figure $8 b$ ).

a

Cancer-associated fibroblasts

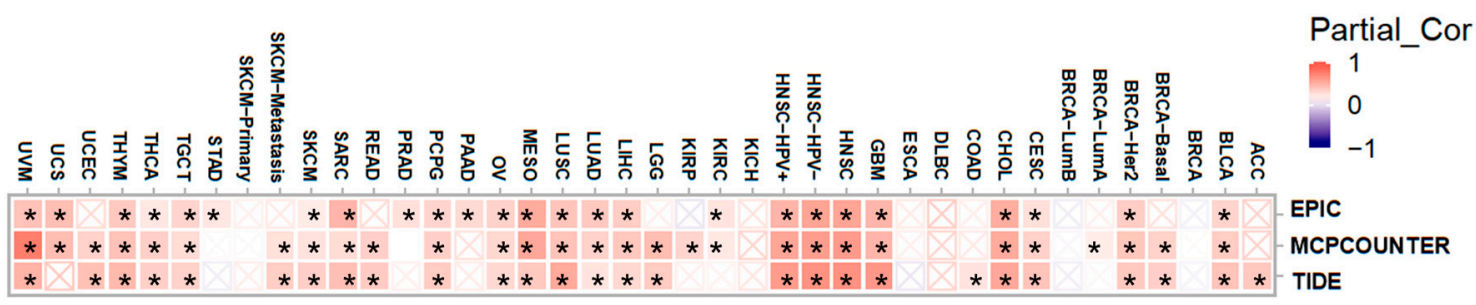

$\mathrm{b}$
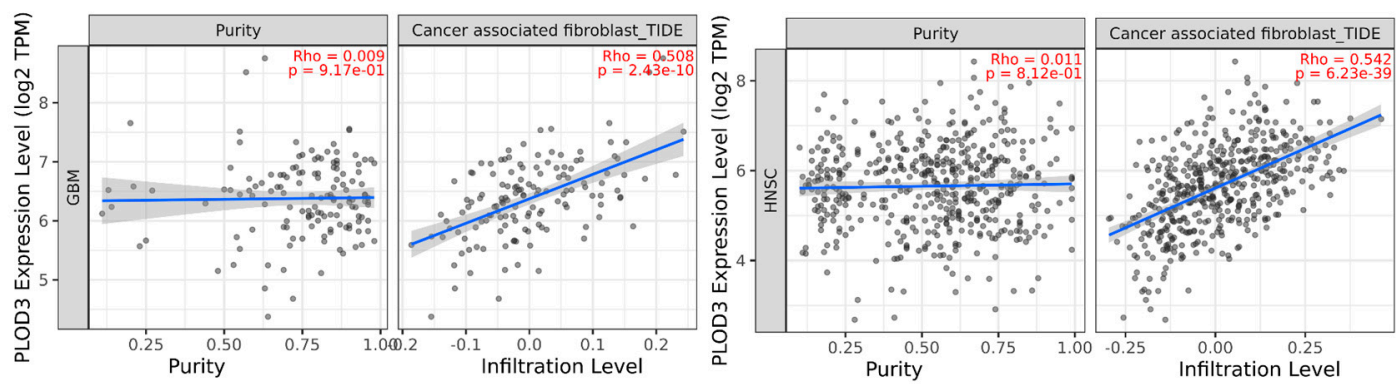

C

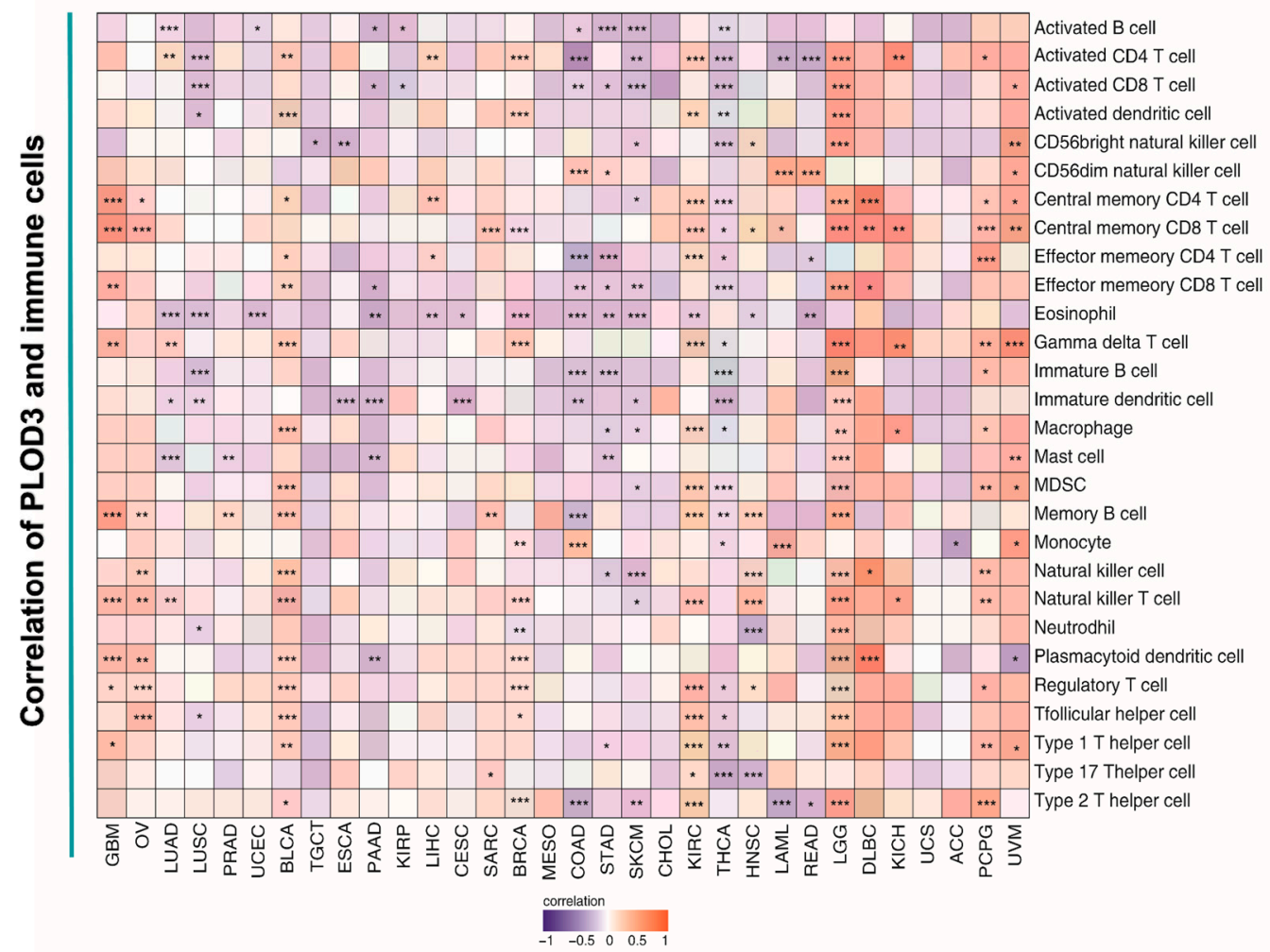

Figure 8. The analysis of relation between PLOD3 expression, immune infiltration of cancer associated fibroblasts and the immune cells. (a) Three kinds of algorithms were used to explore the possible correlation between the expression level of the PLOD3 gene and the infiltration level of cancer associated fibroblasts across serious types of cancer in TCGA; (b) Scatter plots of cancer associated fibroblasts immune infiltration in different tumors generated based on a certain algorithm; (c) Association between PLOD3 expression and different immune cells in different tumors. ${ }^{*} p<0.05,{ }^{* *} p<0.01$, $* * * p<0.001$. 
The Sangerbox tool was used to obtain the immune maps that could show the relation between the expression of PLOD3 and immune cell infiltration in a tumor microenvironment (TME). In total, 28 kinds of immune cells were included in the immune cells map, such as activated $\mathrm{B}$ cell, activated $\mathrm{CD} 4^{+} \mathrm{T}$ cell and activated $\mathrm{CD} 8^{+} \mathrm{T}$ cell. The immune map showed that the expression of PLOD3 in BLCA, KIRC and LGG was positively correlated with most of the immune cells, while THCA was negatively corelated with most of the immune cells. The results are shown in Figure 8c.

In addition, we also investigated the relation of PLOD3 expression to the tumor mutational burden (TMB) and microsatellite instability (MSI), respectively, in different cancers from the TCGA database. The PLOD3 expression is positively related to TMB for KICH, LGG, KIRC, STAD, BRCA, SARC, KIRP, PAAD, ESCA, LUAD and ACC, whereas the expression of PLOD3 is negatively related to TMB for DLBC, LAML and COAD (all $p<0.05)$. The high expression of PLOD3 is associated with high MSI in ACC, MESO, BRCA, SARC, CESC, BLCA and LUSC, but correlates with low MSI in READ and COAD (all $p<0.05$ ) (Figure 9).

\section{a}
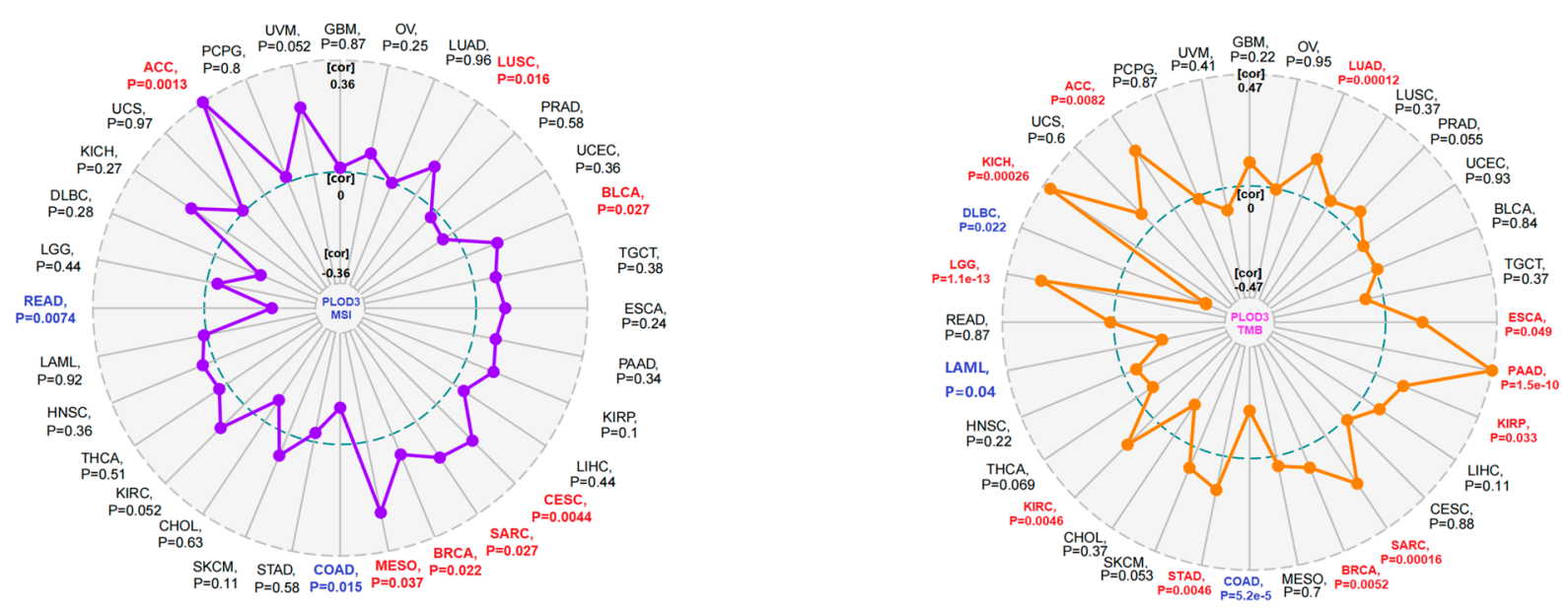

Figure 9. Correlation between PLOD3 expression and microsatellite instability (MSI) or tumor mutational burden (TMB). Based on TCGA database, MSI and TMB were obtained. The $p$-value is supplied. (a) The potential correlation between PLOD3 expression and MSI. The partial correlation (Cor) values of +0.36 and -0.36 are marked; (b) The potential correlation between PLOD3 expression and TMB. The partial correlation (Cor) values of +0.47 and -0.47 are marked.

Supplementary Figure S7a demonstrates the relation between PLOD3 expression level and $\mathrm{CD}^{+} \mathrm{T}$ cell infiltration level via ten algorithms (e.g., TIMER, EPIC and MCPCOUNTER). PLOD3 expression level was negatively correlated with $\mathrm{CD} 8^{+} \mathrm{T}$ cell infiltrate level in PAAD and SKCM on the basis of most of the algorithms $(8 / 10$ and $9 / 10)$. The scatter plots generated according to the strongest correlative algorithms are shown in Supplementary Figure S7b.

\section{Discussion}

The PLOD3 gene is critical for collagen synthesis, thus, mutations are related with disorders of the connective tissue and the development of several tumor entities [1,2,5]. In this study, more than 10,000 samples from the CPTAC, FANTOM5, GEO, GTEx, HPA and TCGA databases were analyzed. Tests were performed for gene alteration, gene expression, survival analysis and immune infiltration. Furthermore, a gene enrichment analysis was carried out, to reveal the potential significance of PLOD3 gene regulation in cancer.

In most of the analyzed cancers, PLOD3 gene expression was significantly higher in tissues of cancer compared to its expression in healthy tissues (Figure 3). The overexpres- 
sion of the PLOD3 gene was further linked to poor clinical prognoses (e.g., ACC, LGG, LIHC, Figure 5). This observation, indicating the cancer-promoting abilities of PLOD3, are in line with previously published studies, suggesting that the expression of PLOD3 could lead to oncogenesis and cancer metastasis in a variety of tumors $[3,7,10,11]$. For example, PLOD3 can promote lung cancer metastasis by regulating STAT3, and PLOD3 silencing can inhibit the proliferation of glioma cells via P21 pathway [12,13]. Analyzing the genetic alteration of PLOD3 in our study, it is observable that the mutation and CNA contribute, to a great extent, to diverse cancers. Previous studies have shown that the mutation of PLOD3 can lead to disorders of connective tissue disease and other diseases $[1,2,14,15]$. In addition, it is generally known that genetic alteration plays a key role in the development of tumors [16,17]. In this study, PLOD3 amplification is underrepresented in cancers such as UCEC and SKCM, while mutations occur most often (7.94\% and 3.9\%). Therefore, one could assume that the mutations of the PLOD3 gene are part of the oncogenesis.

Two possible mechanisms that could account for the observed effects are discussed in the literature. One mechanism relates to the extracellular matrix, a component of the cellular microenvironment that is composed of structural proteins, glycoproteins and proteoglycans. As collagen is one of the main components of the extracellular matrix, it takes part in regulating cancer cell invasion, metastasis and survival through biochemical and biophysical signals, resulting from collagen-cell interactions [15,18-23]. Lysyl Dydroxylase 3 (LH3), a protein highly involved in collagen biosynthesis, is encoded by PLOD3 [1,9,19]. Another possibility is somatcanCNA. Somatic changes influence a great part of the genome in cancer cells, and are involved in many kinds of genetic alteration, such as single base substitutions, translocations and infections, influencing oncogenes and tumor suppressor genes [23,24]. Therefore, understanding the mechanisms resulting in PLOD3 mutation, as well as its biological effects, might affect the diagnosis and therapy of cancer.

The String tool and GeneMANIA were used to reveal a comprehensive network of PLOD3 related genes with physical or functional interactions [25-27]. The 100 most highly correlated genes with PLOD3 were selected (Supplementary Table S1) and the expression of the top five was examined for 33 tumor entities (Figure $6 \mathrm{~b}, \mathrm{c}$ ). Although no regulatory relationships between PLOD3 and the top five interacted genes have been reported in the literature, a potential regulatory network was still identified (Supplementary Figure S8). This may be conducive to further revealing the mechanism of PLOD3 in tumors in the future. In fact, previous studies indicate that ARPC1B is a component of the Arp2/3 complex, a polyprotein composite, which can mediate the formation of branch myocyanin networks in the cytoplasm, providing a driving force of cell motility [28,29]. PARVB is involved in the function of actin and plays a key role in cell adhesion, diffusion and migration, which suggests their potential role in EMT [30]. In contraction, PLOD3 is also closely related to EMT in our previous description, which further supports our conclusion. In addition, it is worth noting that HILS1 is also related to chromatin remodeling and transcriptional regulation [31], in view of its strong correlation with PLOD3 and the result of enrichment analysis, this may imply the potential role of PLOD3 in chromatin function.

In the enrichment study, the KEGG pathway and the GO enrichment analysis especially deserve attention. The KEGG pathway shows that PLOD3 was related with protein digestion and absorption, focal adhesion, and small cell lung cancer, which could be a potential reason for the alteration of PLOD3 in disorders of the connective tissue, tumorigenesis, and cancer progression $[1-4,7,10,11]$, this is worthy of further research. Previous research stated that proteins function as team players to form complex cellular machines and transmit signals within cells [32]. Protein-protein interactions affect cellular pathways in living organisms, thus, deciphering changes in the protein facilitates the understanding of tumorigenesis on a systematic level [32,33]. With respect to the cellular component of $\mathrm{GO}$, the importance of the research on the endoplasmic reticulum lumen is supported by other studies $[34,35]$. High quality protein folding plays a vital role in cell survival and the normal physiology of the organism [35]. However, altered endoplasmic reticulum homeostasis causes the unfolded and misfolded proteins to accumulate in the endoplasmic 
reticulum lumen, which is known as endoplasmic reticulum stress and could facilitate a cell survival mode to restore homeostasis [36,37]. Chronic or severe endoplasmic reticulum stress, however, induces intracellular oxidative stress and impairs the mitochondrial function, which leads to cell apoptosis [37]. According to our enrichment study, PLOD3 related genes are involved in protein related processes, such as "endoplasmic reticulum lumen", "protein folding chaperone" and "misfolded protein binding", which could be one potential mechanism of tumor formation.

Our immunohistochemical analysis of various cancer tissues revealed glial cells and $\mathrm{CAF}$ as the main cellular population expressing PLOD3. CAFs are involved in regulating tumor-infiltrating immune cells, cancer initiation, progression, or metastasis [38-41]. These cells form one of the most necessary factors of the TME, playing a key role in the process of tumor infiltration, in promoting oncogenesis, and are associated with the immune evasion of cancer cells [42-45]. Research has shown that targeting CAF derived factors could be promising in patients with drug resistance to immune checkpoint inhibitors (ICIs) [44]. It is well known that $\mathrm{CD} 8^{+} \mathrm{T}$ cells play a crucial role in the antitumor effect of ICIs [46-49]. However, the $\mathrm{CD}^{+} \mathrm{T}$ cells are mostly in an "exhausted" condition due to prolonged inflammation in cancer $[46,47,49]$. This study shows that PLOD3 expression was negatively correlated to the infiltration degree of $\mathrm{CD}^{+} \mathrm{T}$ cells, but was positively related to CAFs in the following cancers, SKCM, LUSC and THCA (Figure 8). This result suggests that PLOD3 targeted drugs might be beneficial, in combination with immunotherapy, against these cancers. In COAD studies it was shown that CAF could be a factor affecting tumor initiation and enhanced drug resistance $[50,51]$. Some other studies show that CAFs play a role in the process of cancer metastasis and related PLOD3 to metastasis in LUAD [10,52,53].

Further, Wu et al. elucidated that high macrophage and neutrophile infiltration prominently reduced the prognosis of prostate cancer, indicating the possibility of the aforementioned two kinds of cells as drug targets for prostate cancer [41]. In this study, the PLOD3 gene was positively correlated with macrophage and neutrophile infiltration level in LGG, which may indicate that a target drug could be effective in LGG.

Immunotherapy is well implemented in the clinical practice of various cancers nowadays [54-56]. Previous studies have shown that the cytotoxic T lymphocyte associated protein-4 (CTLA-4) and programmed cell death protein-1 (PD-1) play an essential role in the development of cancer immunotherapy [57,58]. Recent research has shown the infiltration of immune cells in cancers and the possibility of these as prognostic biomarkers [40,59]. MSI is a hypermutable phenotype caused by the loss of DNA mismatch repair activity, and is used to predict prognosis of cancers $[60,61]$. TMB is also a biomarker to predict the response to immune target therapy [60-64]. Our study demonstrated that PLOD3 is positively correlated with the TMB in tumors such as LGG and KIRC. The immune map further shows that LGG and KIRC are positively related with most of the immune cells; together these results suggest, again, that targeting PLOD3 might be beneficial for immune therapy (Figure 9). Nevertheless, there are variabilities in certain cancer entities and a multigene analysis should address this limitation. The PLOD3 gene belongs to the PLOD gene family, and the PLOD gene family are related to each other (Supplementary Figure S6). However, using the PLOD gene family and PLOD3 protein expression might be a valuable marker for cancer prognosis.

Nevertheless, no therapy dependent effect could be detected in the current study. Baek et al. proved that PLOD3 knockdown could inhibit renal cell carcinoma malignance as well as lung tumor growth. In lung cancer cells, PLOD3 knockdown suppresses chemoresistance and radioresistance, which might promote the curative effect with chemotherapy and radiotherapy [65-67]. Considering the essential role of PLOD3, and the encoded enzyme, in the formation of the extracellular matrix derived from fibroblasts, chondroblasts and osteoblasts, we were not surprised that it could also be overexpressed in connective tissue malignancies (SARC), compared to corresponding normal tissue, according to the immunohistochemical analyses. Considering the findings of Jiang et al., that PLOD1 is a prognostic marker in osteosarcomas [68]. Further studies, especially of soft tissue sarcomas, 
are pending. In future research, we suggest exploring whether PLOD3 expression relates to the sensitivity of therapeutic interventions, such as radio-, immuno- and chemotherapy across various types of cancers.

Taken together, our findings elucidate that PLOD3 could act as an inducer of various cancers, PLOD3 expression possessed a negative correlation with the survival odds and the infiltration level of $\mathrm{CAF}$ and a negative relation to the $\mathrm{CD} 8^{+} \mathrm{T}$ cells infiltration, to a certain extent. Hence, we may identify PLOD3 as a prognostic biomarker and a potential therapeutic target for some cancers, in an attempt to improve survival probability.

\section{Materials and Methods}

\subsection{Genetic Alteration Analysis}

"Cancer Types Summary" module of the cBioPortal was used to obtain the PLOD3 gene data and we entered the PLOD3 gene in the "TCGA Pan Cancer Atlas Studies" module to obtain the tumor entities summary, alteration frequency and CNA data.

The "mutation" module was chosen to gain the diagram of PLOD3 alteration sites that included the alternation types and number.

\subsection{Gene expression Analysis}

After entry into the tumor immune estimation resource, version 2 (TIMER2) (http: / / timer.cistrome.org/, accessed on 20 May 2021), PLOD3 gene was studied to gain discrepancies in its expression, between cancer and normal tissue, for various cancers or particular subtypes of cancers, according to the data from TCGA database. Then, the gene expression profiling interactive analysis, version 2 (GEPIA2) (http:/ / gepia2.cancer-pku.cn/\#analysis, accessed on 20 May 2021), was employed to analyze the expression of PLOD3 in cancers where only tumors or limited normal cases existed in the last step; hereby, the box plot was obtained for those cancers and their adjacent normal tissues by matched TCGA normal and GTEx data and default of log2FC (fold change) cutoff, $p$-value cutoff. Then, the violin plot was obtained to explore the PLOD3 expression in dissimilar pathological stages for types of cancers from TCGA database. In addition, UALCAN (http:/ / ualcan.path.uab.edu/analysis-prot.html, accessed on 20 May 2021) was used to assess the PLOD3 total protein for cancers such as BRCA, UCEC and LUAD, on the basis of clinical proteomic tumor analysis consortium (CPTAC) dataset.

Moreover, the HPA database (https:/ /www.proteinatlas.org/humanproteome/pathology, accessed on 20 May 2021) was used to obtain the expression data of the PLOD3 gene in various cells and tissues under physiological conditions.

Patient derived cancer tissues were obtained from University Hospital Leipzig. Three different cancers (COAD, GBM and SARC) were chosen in this study. For each entity, tissue samples from three patients were stained by 3,3'-diaminobenzidine tablets (DAB, Sigma Aldrich, St. Louis, MO, USA). Immunohistochemical staining was carried out as described previously [69]. The following antibody were used: PLOD3 antibody (11027-1-AP, Lot 00059310, 1:100 dilution, ProteinTech Group, Chicago, IL, USA).

\subsection{Survival Prognosis Analysis}

Survival maps of PLOD3 were obtained for diverse cancers from the TCGA database through " $50 \%$ cutoff-high and $50 \%$ cutoff-low" on the GEPIA2 to separate into high and low expression groups. Then, "survival analysis" was acquired to evaluate the effect of PLOD3 expression on OS and DFS across different tumors that had statistical significance in last step, herein, the log-rank was applied.

Kaplan-Meier plotter (http:/ / kmplot.com/analysis/, accessed on 25 May 2021) was used to analyze the OS, distant metastasis free, relapse free, postprogression, disease specific, and progress free survival, and first progression of lung, ovarian, gastric, and liver cancers based on "auto select best cutoff". 


\subsection{Construction of Related Genes Network}

The STRING (https://string-db.org/, accessed on 25 May 2021) was employed to determine the PLOD3 interacted proteins with input PLOD3 and the application of following parameters: "evidence" for "meaning of network edges", "experiments" for "active interaction sources" and "disable structure previews inside network bubbles" for "network display options". GeneMania (https://genemania.org/, accessed on 15 August 2021) was also used to further construct the interactive network of the top 5 PLOD3 interacted proteins. Then, the top 100 PLOD3 related genes were acquired on the "similar genes detection" module of GEPIA2. Picked genes were, respectively, used to conduct a dot plot for the correlation analysis through the application of Pearson correlation and log2 TPM and the mark of $p$-value and the correlation coefficient (R). In addition, the chosen genes were entered into the "Gene_Corr" module of TIMER2 to perform a heatmap for the correlation analysis with PLOD3 for all TCGA database cancers based on the purity adjustment and indication of the partial correlation (cor) and $p$-value. In addition, the GeneMANIA tool (https://genemania.org/, accessed on 15 August 2021) was used to obtain the network of PLOD3 and related genes.

\subsection{KEGG Pathway and GO Enrichment Analysis}

The data of PLOD3 related genes were combined to KEGG pathway and GO analysis with following method: R package "clusterProfiler" in R software (Version 4.0.3, R Foundation for Statistical Computing, Vienna, Austria) was used to perform KEGG pathway and GO enrichment analysis including biological process, cellular component, and molecular function.

\subsection{Immune Infiltration Analysis}

"PLOD3" and "cancer associated fibroblast" were entered on the "immune-gene" of TIMER2 to obtain a heatmap about the correlation analysis between PLOD3 gene expression and CAF across all cancers in TCGA database on the basis of purity adjustment and three algorithms, namely, EPIC, MCP-COUNTER and TIDE, in this process the negative or positive correlation were shown with the $p$-values and correlation values. Then, the scatter plots were acquired to assess the specific correlation condition for the cancers where PLOD3 possessed positive correlation with the cancer associated fibroblast via all three algorithms based on the last step.

The relation between the PLOD3 gene expression and the $\mathrm{CD}^{+} \mathrm{T}$ cells infiltration was analyzed via the "Immune-Gene" of TIMER2 based on some algorithms (e.g., TIMER, EPIC, MCPCOUNTER) and the scatter plots were acquired for some selected cancers.

PLOD3 was put in the Sangerbox tool (http://sangerbox.com/Tool, accessed on 30 May 2021) to explore the potent relation of the PLOD3 expression to TMB or MSI across different cancers from TCGA database. Furthermore, the relationship between PLOD3 expression and immune cells was also explored.

Supplementary Materials: The following are available online at https:/ / www.mdpi.com/article/10 $.3390 /$ ijms22189903/s1.

Author Contributions: Conception and design: S.G., N.S., C.W. and S.K. Collection and assembly of data: S.G., Y.D. and C.W. Data analysis and interpretation: S.G., C.W., G.O., N.S. and S.K. Manuscript writing and revisions: S.G., N.S., G.O. and S.K. Final approval of manuscript: all authors. Accountable for all aspects of work: all authors. All authors have read and agreed to the published version of the manuscript.

Funding: This research received no external funding. However, we acknowledge support from Leipzig University for Open Access Publishing.

Institutional Review Board Statement: The examinations of human tissue samples were approved by the Ethical Committee at the Medical Faculty, Leipzig University (AZ 370-1316122013, 3 December 2018). 
Informed Consent Statement: Informed consent was obtained from all subjects involved in the study.

Data Availability Statement: The data provided in this study can be obtained in the method section of this manuscript. The results shown here are, in part, based upon data generated by TCGA Research Network (https:/ / www.cancer.gov/tcga), GTEx database (https://commonfund.nih.gov/gtex) and HPA database (https:/ / www.proteinatlas.org/humanproteome/pathology).

Conflicts of Interest: The authors declare that they have no competing interests. The funders had no role in the design of the study; in the collection, analyses, or interpretation of data; in the writing of the manuscript, or in the decision to publish the results.

\section{References}

1. Salo, A.M.; Cox, H.; Farndon, P.; Moss, C.; Grindulis, H.; Risteli, M.; Robins, S.P.; Myllylä, R. A Connective Tissue Disorder Caused by Mutations of the Lysyl Hydroxylase 3 Gene. Am. J. Hum. Genet. 2008, 83, 495-503. [CrossRef] [PubMed]

2. Vahidnezhad, H.; Youssefian, L.; Saeidian, A.H.; Touati, A.; Pajouhanfar, S.; Baghdadi, T.; Shadmehri, A.A.; Giunta, C.; Kraenzlin, M.; Syx, D.; et al. Mutations in PLOD3, encoding lysyl hydroxylase 3, cause a complex connective tissue disorder including recessive dystrophic epidermolysis bullosa-like blistering phenotype with abnormal anchoring fibrils and type VII collagen deficiency. Matrix Biol. 2019, 81, 91-106. [CrossRef]

3. Li, S.S.; Lian, Y.F.; Huang, Y.L.; Huang, Y.H.; Xiao, J. Overexpressing PLOD family genes predict poor prognosis in gastric cancer. J. Cancer 2020, 11, 121-131. [CrossRef] [PubMed]

4. Heikkinen, J.; Hautala, T.; Kivirikko, K.I.; Myllylä, R. Structure and Expression of the Human Lysyl Hydroxylase Gene (PLOD): Introns 9 and 16 Contain Alu Sequences at the Sites of Recombination in Ehlers-Danlos Syndrome Type VI Patients. Genomics 1994, 24, 464-471. [CrossRef] [PubMed]

5. Schneider, V.A.; Granato, M. Genomic structure and embryonic expression of zebrafish lysyl hydroxylase 1 and lysyl hydroxylase 2. Matrix Biol. 2007, 26, 12-19. [CrossRef]

6. Scietti, L.; Campioni, M.; Forneris, F. SiMPLOD, a Structure-Integrated Database of Collagen Lysyl Hydroxylase (LH/PLOD) Enzyme Variants. J. Bone Miner. Res. 2019, 34, 1376-1382. [CrossRef]

7. Wang, B.; Xu, L.; Ge, Y.; Cai, X.; Li, Q.; Yu, Z.; Wang, J.; Wang, Y.; Lu, C.; Wang, D.; et al. PLOD3 is Upregulated in Gastric Cancer and Correlated with Clinicopathologic Characteristics. Clin. Lab. 2019, 65, 25-30. [CrossRef]

8. Stone, R.C.; Pastar, I.; Ojeh, N.; Chen, V.; Liu, S.; Garzon, K.I.; Tomic-Canic, M. Epithelial-mesenchymal transition in tissue repair and fibrosis. Cell Tissue Res. 2016, 365, 495-506. [CrossRef]

9. Lee, C.H. Reversal of Epithelial-Mesenchymal Transition by Natural Anti-Inflammatory and Pro-Resolving Lipids. Cancers 2019, 11, 1841. [CrossRef]

10. Roversi, G.; Pfundt, R.; Moroni, R.F.; Magnani, I.; Van Reijmersdal, S.; Pollo, B.; Straatman, H.; Larizza, L.; Schoenmakers, E.F.P.M. Identification of novel genomic markers related to progression to glioblastoma through genomic profiling of 25 primary glioma cell lines. Oncogene 2006, 25, 1571-1583. [CrossRef]

11. Hui, D.; Paiva, C.E.; Del Fabbro, E.G.; Steer, C.; Naberhuis, J.; Van De Wetering, M.; Fernandez-Ortega, P.; Morita, T.; Suh, S.Y.; Bruera, E.; et al. Prognostication in advanced cancer: Update and directions for future research. Support. Care Cancer 2019, 27, 1973-1984. [CrossRef]

12. Baek, J.H.; Yun, H.S.; Kwon, G.T.; Kim, J.Y.; Lee, C.W.; Song, J.Y.; Um, H.D.; Kang, C.M.; Park, J.K.; Kim, J.S.; et al. PLOD3 promotes lung metastasis via regulation of STAT3. Cell Death Dis. 2018, 9, 1138. [CrossRef]

13. Tsai, C.K.; Huang, L.C.; Tsai, W.C.; Huang, S.M.; Lee, J.T.; Hueng, D.Y. Overexpression of PLOD3 promotes tumor progression and poor prognosis in gliomas. Oncotarget 2018, 9, 15705-15720. [CrossRef]

14. Saxena, A.K.; Tiwari, M.; Agarwal, M.; Kumar, A.A. Prediction of 3D Protein Structure Based on The Mutation of AKAP3 and PLOD3 Genes in The Case of Non-Obstructive Azoospermia. Int. J. Fertil. Steril. 2020, 14, 102-109. [CrossRef]

15. Ewans, L.J.; Colley, A.; Gaston-Massuet, C.; Gualtieri, A.; Cowley, M.J.; McCabe, M.J.; Anand, D.; Lachke, S.A.; Scietti, L.; Forneris, F.; et al. Pathogenic variants in PLOD3 result in a Stickler syndrome-like connective tissue disorder with vascular complications. J. Med. Genet. 2019, 56, 629-638. [CrossRef]

16. Hanahan, D.; Weinberg, R.A. Hallmarks of Cancer: The Next Generation. Cell 2011, 144, 646-674. [CrossRef] [PubMed]

17. Balmain, A. The critical roles of somatic mutations and environmental tumor-promoting agents in cancer risk. Nat. Genet. 2020, 52, 1139-1143. [CrossRef] [PubMed]

18. Visconti, L.; Nelissen, K.; Deckx, L.; Van Den Akker, M.; Adriaensen, W.; Daniels, L.; Matheï, C.; Linsen, L.; Hellings, N.; Stinissen, P.; et al. Prognostic value of circulating cytokines on overall survival and disease-free survival in cancer patients. Biomark. Med. 2014, 8, 297-306. [CrossRef]

19. Qi, Y.; Xu, R. Roles of PLODs in Collagen Synthesis and Cancer Progression. Front. Cell Dev. Biol. 2018, 6, 66. [CrossRef] [PubMed]

20. Xiong, G.; Deng, L.; Zhu, J.; Rychahou, P.G.; Xu, R. Prolyl-4-hydroxylase $\alpha$ subunit 2 promotes breast cancer progression and metastasis by regulating collagen deposition. BMC Cancer 2014, 14, 1. [CrossRef]

21. Pollard, J.W. Tumour-educated macrophages promote tumour progression and metastasis. Nat. Rev. Cancer 2004, 4, 71-78. [CrossRef] [PubMed] 
22. Oudin, M.J.; Jonas, O.; Kosciuk, T.; Broye, L.C.; Guido, B.C.; Wyckoff, J.; Riquelme, D.; Lamar, J.M.; Asokan, S.B.; Whittaker, C.; et al. Tumor Cell-Driven Extracellular Matrix Remodeling Drives Haptotaxis during Metastatic Progression. Cancer Discov. 2016, 6, 516-531. [CrossRef]

23. Cheon, D.J.; Tong, Y.; Sim, M.S.; Dering, J.; Berel, D.; Cui, X.; Lester, J.; Beach, J.A.; Tighiouart, M.; Walts, A.E.; et al. A CollagenRemodeling Gene Signature Regulated by TGF- $\beta$ Signaling Is Associated with Metastasis and Poor Survival in Serous Ovarian Cancer. Clin. Cancer Res. 2014, 20, 711-723. [CrossRef] [PubMed]

24. Zack, T.I.; Schumacher, S.E.; Carter, S.L.; Cherniack, A.D.; Saksena, G.; Tabak, B.; Lawrence, M.S.; Zhang, C.-Z.; Wala, J.; Mermel, C.H.; et al. Pan-cancer patterns of somatic copy number alteration. Nat. Genet. 2013, 45, 1134-1140. [CrossRef]

25. Szklarczyk, D.; Gable, A.L.; Lyon, D.; Junge, A.; Wyder, S.; Huerta-Cepas, J.; Simonovic, M.; Doncheva, N.T.; Morris, J.H.; Bork, P.; et al. STRING v11: Protein-protein association networks with increased coverage, supporting functional discovery in genome-wide experimental datasets. Nucleic Acids Res. 2019, 47, D607-D613. [CrossRef] [PubMed]

26. Szklarczyk, D.; Gable, A.L.; Nastou, K.C.; Lyon, D.; Kirsch, R.; Pyysalo, S.; Doncheva, N.T.; Legeay, M.; Fang, T.; Bork, P.; et al. The STRING database in 2021: Customizable protein-protein networks, and functional characterization of user-uploaded gene/measurement sets. Nucleic Acids Res. 2021, 49, D605-D612. [CrossRef]

27. Warde-Farley, D.; Donaldson, S.L.; Comes, O.; Zuberi, K.; Badrawi, R.; Chao, P.; Franz, M.; Grouios, C.; Kazi, F.; Lopes, C.T.; et al. The GeneMANIA prediction server: Biological network integration for gene prioritization and predicting gene function. Nucleic Acids Res. 2010, 38, W214-W220. [CrossRef]

28. Gournier, H.; Goley, E.D.; Niederstrasser, H.; Trinh, T.; Welch, M.D. Reconstitution of Human Arp2/3 Complex Reveals Critical Roles of Individual Subunits in Complex Structure and Activity. Mol. Cell 2001, 8, 1041-1052. [CrossRef]

29. Welch, M.D.; DePace, A.H.; Verma, S.; Iwamatsu, A.; Mitchison, T.J. The Human Arp2/3 Complex Is Composed of Evolutionarily Conserved Subunits and Is Localized to Cellular Regions of Dynamic Actin Filament Assembly. J. Cell Biol. 1997, 138, 375-384. [CrossRef]

30. Matsuda, C.; Kameyama, K.; Suzuki, A.; Mishima, W.; Yamaji, S.; Okamoto, H.; Nishino, I.; Hayashi, Y.K. Affixin activates Rac1 via $\beta$ PIX in C2C12 myoblast. FEBS Lett. 2008, 582, 1189-1196. [CrossRef]

31. Ye, X.; Feng, C.; Gao, T.; Mu, G.; Zhu, W.; Yang, Y. Linker Histone in Diseases. Int. J. Biol. Sci. 2017, 13, 1008-1018. [CrossRef] [PubMed]

32. Gulfidan, G.; Turanli, B.; Beklen, H.; Sinha, R.; Arga, K.Y. Pan-cancer mapping of differential protein-protein interactions. Sci. Rep. 2020, 10, 1-12. [CrossRef]

33. Yeger-Lotem, E.; Sharan, R. Human protein interaction networks across tissues and diseases. Front. Genet. 2015, 6, 257. [CrossRef] [PubMed]

34. Patil, C.; Walter, P. Intracellular signaling from the endoplasmic reticulum to the nucleus: The unfolded protein response in yeast and mammals. Curr. Opin. Cell Biol. 2001, 13, 349-355. [CrossRef]

35. Kaufman, R.J. Stress signaling from the lumen of the endoplasmic reticulum: Coordination of gene transcriptional and translational controls. Genes Dev. 1999, 13, 1211-1233. [CrossRef]

36. Schröder, M.; Kaufman, R.J. The mammalian unfolded protein response. Annu. Rev. Biochem. 2005, 74, 739-789. [CrossRef]

37. Wang, M.; Kaufman, R.J. Protein misfolding in the endoplasmic reticulum as a conduit to human disease. Nature 2016, 529, 326-335. [CrossRef]

38. Wu, C.; Duan, Y.; Gong, S.; Kallendrusch, S.; Schopow, N.; Osterhoff, G. Integrative and Comprehensive Pancancer Analysis of Regulator of Chromatin Condensation 1 (RCC1). Int. J. Mol. Sci. 2021, 22, 7374. [CrossRef]

39. Fridman, W.H.; Galon, J.; Dieu-Nosjean, M.-C.; Cremer, I.; Fisson, S.; Damotte, D.; Pagès, F.; Tartour, E.; Sautès-Fridman, C. Immune Infiltration in Human Cancer: Prognostic Significance and Disease Control. Curr. Top. Microbiol. Immunol. 2010, 344, 1-24. [CrossRef]

40. Fu, J.; Yu, A.; Tang, J.; He, B.; Chen, W. Adoptive CD8 + T cell therapy generates immunological memory to inhibit melanoma metastasis. Am. J. Transl. Res. 2020, 12, 7262-7274. [PubMed]

41. Wu, Z.; Chen, H.; Luo, W.; Zhang, H.; Li, G.; Zeng, F.; Deng, F. The Landscape of Immune Cells Infiltrating in Prostate Cancer. Front. Oncol. 2020, 10, 517637. [CrossRef] [PubMed]

42. Steven, A.; Seliger, B. The Role of Immune Escape and Immune Cell Infiltration in Breast Cancer. Breast Care 2018, 13, 16-21. [CrossRef] [PubMed]

43. Chen, X.; Song, E. Turning foes to friends: Targeting cancer-associated fibroblasts. Nat. Rev. Drug Discov. 2019, 18, 99-115. [CrossRef] [PubMed]

44. Kwa, M.Q.; Herum, K.M.; Brakebusch, C. Cancer-associated fibroblasts: How do they contribute to metastasis? Clin. Exp. Metastasis 2019, 36, 71-86. [CrossRef] [PubMed]

45. Liu, T.; Han, C.; Wang, S.; Fang, P.; Ma, Z.; Xu, L.; Yin, R. Cancer-associated fibroblasts: An emerging target of anti-cancer immunotherapy. J. Hematol. Oncol. 2019, 12, 86. [CrossRef] [PubMed]

46. He, Q.F.; Xu, Y.; Li, J.; Huang, Z.M.; Li, X.H.; Wang, X. CD81 T-cell exhaustion in cancer: Mechanisms and new area for cancer immunotherapy. Brief. Funct. Genom. 2019, 18, 99-106. [CrossRef]

47. Zhang, L.; Romero, P. Metabolic Control of CD8+ T Cell Fate Decisions and Antitumor Immunity. Trends Mol. Med. 2018, 24, 30-48. [CrossRef] 
48. Farhood, B.; Najafi, M.; Mortezaee, K. CD8(+) cytotoxic T lymphocytes in cancer immunotherapy: A review. J. Cell. Physiol. 2019, 234, 8509-8521. [CrossRef]

49. Blank, C.U.; Haining, W.N.; Held, W.; Hogan, P.G.; Kallies, A.; Lugli, E.; Lynn, R.C.; Philip, M.; Rao, A.; Restifo, N.P.; et al. Defining 'T cell exhaustion'. Nat. Rev. Immunol. 2019, 19, 665-674. [CrossRef]

50. Uchihara, T.; Miyake, K.; Yonemura, A.; Komohara, Y.; Itoyama, R.; Koiwa, M.; Yasuda, T.; Arima, K.; Harada, K.; Eto, K.; et al. Extracellular Vesicles from Cancer-Associated Fibroblasts Containing Annexin A6 Induces FAK-YAP Activation by Stabilizing b1 Integrin, Enhancing Drug Resistance. Cancer Res. 2020, 80, 3222-3235. [CrossRef]

51. Seton-Rogers, S. Fibroblasts orchestrate tumour initiation. Nat. Rev. Cancer 2020, 20, 301. [CrossRef] [PubMed]

52. Pein, M.; Insua-Rodríguez, J.; Hongu, T.; Riedel, A.; Meier, J.; Wiedmann, L.; Decker, K.; Essers, M.A.G.; Sinn, H.P.; Spaich, S.; et al. Metastasis-initiating cells induce and exploit a fibroblast niche to fuel malignant colonization of the lungs. Nat. Commun. 2020, 11, 1494. [CrossRef] [PubMed]

53. Eckert, M.A.; Coscia, F.; Chryplewicz, A.; Chang, J.W.; Hernandez, K.M.; Pan, S.; Tienda, S.M.; Nahotko, D.A.; Li, G.; Blaženović, I.; et al. Proteomics reveals NNMT as a master metabolic regulator of cancer-associated fibroblasts. Nature 2019, 569, 723-728. [CrossRef]

54. Zhang, Y.; Zhang, Z. The history and advances in cancer immunotherapy: Understanding the characteristics of tumor-infiltrating immune cells and their therapeutic implications. Cell. Mol. Immunol. 2020, 17, 807-821. [CrossRef]

55. Murciano-Goroff, Y.R.; Warner, A.B.; Wolchok, J.D. The future of cancer immunotherapy: Microenvironment-targeting combinations. Cell Res. 2020, 30, 507-519. [CrossRef]

56. Mattox, A.K.; Lee, J.; Westra, W.H.; Pierce, R.H.; Ghossein, R.; Faquin, W.C.; Diefenbach, T.J.; Morris, L.G.; Lin, D.T.; Wirth, L.J.; et al. PD-1 Expression in Head and Neck Squamous Cell Carcinomas Derives Primarily from Functionally Anergic CD4+ TILs in the Presence of PD-L1+ TAMs. Cancer Res. 2017, 77, 6365-6374. [CrossRef]

57. Haanen, J.B.A.G.; Robert, C. Immune Checkpoint Inhibitors. Prog. Tumor Res. 2015, 42, 55-66.

58. Syn, N.L.; Teng, M.W.L.; Mok, T.S.K.; Soo, R.A. De-novo and acquired resistance to immune checkpoint targeting. Lancet Oncol. 2017, 18, e731-e741. [CrossRef]

59. Yang, B.; Zhao, Y.; Wang, L.; Zhao, Y.; Wei, L.; Chen, D.; Chen, Z. Identification of PLOD Family Genes as Novel Prognostic Biomarkers for Hepatocellular Carcinoma. Front. Oncol. 2020, 10, 1695. [CrossRef] [PubMed]

60. Boland, C.R.; Goel, A. Microsatellite instability in colorectal cancer. Gastroenterology 2010, 138, 2073-2087.e3. [CrossRef]

61. Lin, A.; Zhang, J.; Luo, P. Crosstalk Between the MSI Status and Tumor Microenvironment in Colorectal Cancer. Front. Immunol. 2020, 11, 2039. [CrossRef]

62. Postow, M.A.; Callahan, M.K.; Wolchok, J.D. Immune Checkpoint Blockade in Cancer Therapy. J. Clin. Oncol. 2015, 33, 1974-1982. [CrossRef]

63. Shekarian, T.; Valsesia-Wittmann, S.; Brody, J.; Michallet, M.C.; Depil, S.; Caux, C.; Marabelle, A. Pattern recognition receptors: Immune targets to enhance cancer immunotherapy. Ann. Oncol. 2017, 28, 1756-1766. [CrossRef] [PubMed]

64. Liu, X.; Yao, J.; Song, L.; Zhang, S.; Huang, T.; Li, Y. Local and abscopal responses in advanced intrahepatic cholangiocarcinoma with low TMB, MSS, pMMR and negative PD-L1 expression following combined therapy of SBRT with PD-1 blockade. J. Immunother. Cancer 2019, 7, 204. [CrossRef]

65. Schrock, A.B.; Ouyang, C.; Sandhu, J.; Sokol, E.; Jin, D.; Ross, J.S.; Miller, V.A.; Lim, D.; Amanam, I.; Chao, J.; et al. Tumor mutational burden is predictive of response to immune checkpoint inhibitors in MSI-high metastatic colorectal cancer. Ann. Oncol. 2019, 30, 1096-1103. [CrossRef] [PubMed]

66. Baek, J.H.; Yun, H.S.; Kwon, G.T.; Lee, J.; Kim, J.Y.; Jo, Y.; Cho, J.M.; Lee, C.W.; Song, J.Y.; Ahn, J.; et al. PLOD3 suppression exerts an anti-tumor effect on human lung cancer cells by modulating the PKC-delta signaling pathway. Cell Death Dis. 2019, 10, 1-13. [CrossRef] [PubMed]

67. Xie, D.; Li, J.; Wei, S.; Qi, P.; Ji, H.; Su, J.; Du, N.; Zhang, X. Knockdown of PLOD3 suppresses the malignant progression of renal cell carcinoma via reducing TWIST1 expression. Mol. Cell. Probes 2020, 53, 101608. [CrossRef]

68. Jiang, H.; Guo, W.; Yuan, S.; Song, L. PLOD1 Is a Prognostic Biomarker and Mediator of Proliferation and Invasion in Osteosarcoma. BioMed Res. Int. 2020, 2020, 1-12. [CrossRef]

69. Brückner, L.; Reinshagen, A.; Hoang, N.A.; Höhn, A.K.; Lordick, F.; Bechmann, I.; Aktas, B.; Nel, I.; Kallendrusch, S. FOXM1 Inhibition in Ovarian Cancer Tissue Cultures Affects Individual Treatment Susceptibility Ex Vivo. Cancers 2021, 13, 956. [CrossRef] 